\title{
Development of a High-dorsiflexion Assistive System for Subacute Stroke Patients: Intervention Experiment on Healthy Subjects With Dorsiflexion Movement Restriction
}

\author{
Jing-Chen Hong ( $\nabla$ charles4543@toki.waseda.jp ) \\ Waseda University https://orcid.org/0000-0002-4045-087X \\ Hao Cheng \\ Waseda University: Waseda Daigaku \\ Hiroki Ohashi \\ Jikei University School of Medicine: Tokyo Jikeikai Ika Daigaku \\ Hiroyasu Iwata \\ Waseda University: Waseda Daigaku
}

\section{Research Article}

Keywords: Stroke, Hemiplegia, Rehabilitation, Dorsiflexion, Ankle-foot orthosis

Posted Date: September 15th, 2020

DOl: https://doi.org/10.21203/rs.3.rs-73089/v1

License: (c) (1) This work is licensed under a Creative Commons Attribution 4.0 International License.

Read Full License 


\section{Abstract}

A number of robotic ankle-foot orthoses with practical application for stroke patients have been developed in recent years. However, targets are mainly patients in the chronic phase. In this paper, we present development of a high-dorsiflexion assistive system aiming to support passive dorsiflexion movement in the swing phase for subacute patients in the early phase of gait rehabilitation. By applying a McKibben-type artificial muscle, output of high dorsiflexion torque with a low-weighted prototype was realized. This is expected to minimize loading burden on subacute stroke patients. An experiment on six healthy participants was conducted, and different extents of compensatory movements were applied when their dorsiflexion movements were restricted. The results of processed surface electromyography data significantly decreased when dorsiflexion movement was assisted with our system. Meanwhile, the results of spatial parameters also showed significant improvement of compensatory movement inclination with sufficient assistance. These indicate the potential of realizing assistance of passive ankle movements for patients with very low dorsiflexion abilities, which shows the potential for our future planning of practical pilot studies.

\section{Introduction}

A crucial issue of exploding requirements of gait rehabilitation has been focused on in recent decades due to a rapid rise in stroke attack number [1,2]. Hemiplegia is one of the most common sequelae for stroke survivors, and it leads to equinus foot on the paralyzed side [3, 4]. Patients with equinus foot perform toe-down posture in the swing phase in gait, which increases the risk of stumbling. Compensatory movements such as pelvic obliquity and excess hip flexion might be utilized by patients to ensure sufficient minimum toe clearance (MTC) [5-7]. This results in incorrect gait learning during rehabilitation.

In recent years, robotic ankle-foot orthosis (RAFO) has been developed to assist ankle movements during gait rehabilitation, including dorsiflexion in the swing phase [8]. One example is the soft exosuit, which provides ankle assistance in walking with a cable-driven mechanism [9]. The other examples are RAFO devices, which have already been employed in clinical facilities, such as RE-Gait and Cocoroe [10, 11]. These studies provided the potential to improve the ankle gait of stroke survivors with robotic technologies. However, targets of current RAFO studies were mostly limited to patients in the late chronic stage of recovery. To prevent the risk of stumbling due to decreased MTC and incorrect gait learning resulting from compensatory movements, we claim that active ankle assistance in early gait rehabilitation is indispensable.

To assist early gait rehabilitation, a light-weighted device that provides high torque for passive ankle dorsiflexion movement is required. Due to the weights of electric motors and hardware prototypes, the abovementioned related RAFOs were either above $1 \mathrm{~kg}$ for waist or lower-limb parts [8-11]. Extra load, especially on a stroke hemiplegic patient's paralyzed lower limb, might affect their gait pattern during rehabilitation [12]. The characteristic of high torque-to-weight ratio has made McKibben-type artificial 
muscle a recent preference for developing rehabilitation robotics [13]. One example of a pneumatically powered orthosis developed by Ferris et al. assists ankle dorsiflexion and plantarflexion [14]. Another example of a bioinspired soft RAFO developed by Park et al. was even developed without a rigid prototype [15]. Although 4 artificial muscles were applied for assisting ankle movements, the weight of the entire prototype, excluding the air source, was only $950 \mathrm{~g}$, and the dorsiflexion output torque could be as large as $110 \mathrm{Nm}$.

Despite such an advantage, reports of clinical applications with RAFOs powered by pneumatic artificial muscles are still very limited. Because of the high nonlinearity, current studies of artificial muscles are still focused on improving position control accuracy with antagonistic structures and complicated control algorithms [13, 16-18]. For assisting dorsiflexion movement in the swing phase, however, the main goal is to support a high dorsiflexion angle to ensure sufficient MTC [19]. We believe that a simple event-triggered control assisting dorsiflexion in the swing phase is adequate for early gait rehabilitation.

In this research, we aim to develop a high-dorsiflexion assistive system revised from the previous prototype $[20,21]$. It is low-weighted and provides high dorsiflexion assistive torque with a simple eventtriggered control method targeting stroke patients in the early stage of gait rehabilitation. A McKibbentype artificial muscle was applied to lift up the forefoot upon swing phase. An intervention test was conducted on 6 healthy participants with a dorsiflexion restriction orthosis that enables simulation of compensation movements. Dorsiflexion angle in the swing phase, surface electromyography (sEMG), MTC, and evaluation indices related to compensation movements were measured and analyzed to verify the effect of our high-dorsiflexion assistive robotic orthosis. The results indicated our system's capability of providing sufficient assistance for passive dorsiflexion movement during gait rehabilitation and improving compensation movements.

\section{Methods}

The present research aims to develop a high-dorsiflexion assistive robotic system and to verify the potential of the system to be applied in assisting gait rehabilitation with intervention experiments on healthy participants with dorsiflexion movement restriction.

\section{Requirements of the High-dorsiflexion Assistive Robotic System}

The main requirements for the system were set as follows:

1. The weight of the lower-limb part should not be higher than $0.45 \mathrm{~kg}$, and the weight of the waist part should not be higher than $1 \mathrm{~kg}$.

2. The maximum output torque for ankle dorsiflexion assistance should be at least $25 \mathrm{Nm}$.

3. The setup time on a user should be shorter than 3 minutes.

For our previous prototype, the weight of the lower-limb part was $0.56 \mathrm{~kg}$ and that of the waist part was close to $2 \mathrm{~kg}$ [20]. A pilot study on 6 stroke patients was conducted, and the potential for improving 
dorsiflexion movement was indicated. However, identical to other studies, the participants were chronic phase patients. Although no negative effects resulting from the weight of lower-limb part were reported from our previous study, the weight requirement was set even lower as $1 \%$ of an adult's weight. Assuming an adult could be as light as $45 \mathrm{~kg}$, the maximum weight of the lower-limb part was set as $0.45 \mathrm{~kg}$. Meanwhile, most related RAFO devices and our previous prototype deployed the control unit at the waist band or pocket to avoid excess load on the lower-limbs. Conversely, the weights at waist parts are all at least $1.0 \mathrm{~kg}$, which might also be a burden for subacute patients. Therefore, a requirement of a maximum $1.0-\mathrm{kg}$ waist part is crucial.

A previous study reported that an external output torque of approximately $13.5 \mathrm{Nm}$ was required to assist dorsiflexion movement from a drop foot posture [22]. Considering factors of individual differences and our application for subacute patients for full dorsiflexion assistance during gait rehabilitation, the output torque of the system was set close to double, at $25 \mathrm{Nm}$.

Last, the setup time on the user was shorter, and the physical and mental burden on patients was lower, especially for those in the subacute phase. Thus, an extremely short setup time of 3 minutes was set, including putting the system on the users, calibration of the sensor unit, and assistance dorsiflexion angle of the system.

\section{System Design}

The system assists dorsiflexion movement upon the swing phase with contraction of a McKibben-type artificial muscle aligned between the knee and forefoot. The whole system is shown in Fig. 1. The weight of the lower-limb part is $0.35 \mathrm{~kg}$, and that of the waist part is approximately $1.0 \mathrm{~kg}$, which barely fulfills the weight requirement set in the previous session.

Insert Figure 1

\section{A. Lower-limb Part}

The lower-limb part consists of a pressure sensor (FSR-402, Interlink Electronics, USA), an artificial muscle (DMSP-10, Festo Inc., Germany), and a tension spring. The pressure sensor was attached at the inner part of the forefoot. The pressure sensor was deployed at the inner side of the forefoot, which is applied to measure the pressure of the first metatarsal point. Details of the control method with pressure data were explained in Session C. The artificial muscle was fixed on a knee pad, and the tension spring is aligned with the artificial muscle between the knee and forefoot. The spring was applied to support heel rocker function during the loading response phase of gait, and the details were reported in research on the previous prototype [21].

A simplified shank model, depicted in Fig. 2, is applied to check whether the assisted dorsiflexion torque matches the main requirement set in the previous section. Displacement of the tension spring during the swing phase is small enough to be neglected. The equation of assisted dorsiflexion torque is 


$$
\vec{F} \times \overrightarrow{L_{2}}=\vec{\tau}
$$

where $F$ is the assisted force from the artificial muscle, and $L_{2}$ is the length between the ankle joint and the fixed point of the forefoot. The angle $\varphi$ between the foot and $\vec{F}$ is

$$
\varphi=\cos ^{-1}\left(\frac{L_{1}{ }^{2}+L_{2}{ }^{2}-H^{2}}{2 \times L_{1} \times L_{2}}\right)
$$

where $H$ is the length between the knee and ankle joints. Meanwhile, the length between fixed points of the knee and forefoot $L_{1}$ is

$$
L_{1}=\sqrt{L_{2}{ }^{2}+H^{2}-2 \times L_{2} \times H \times \cos \theta}
$$

where $\theta$ is the ankle joint angle. Based on (2) and (3), we can rewrite (1) into

$$
F=\frac{\tau}{L_{2} \times \sin \varphi}
$$

According to research on the physical parameter measurements of stroke patients aged 50 to 99 years old in Japan [23], was set as $0.4 \mathrm{~m}$. was set as $0.15 \mathrm{~m}$ from the measurement of the length between the ankle joint to the forefoot attachment point. was set as due to a plantarflexion angle at the pre-swing phase of gait, assuming is close to at neutral posture [19]. The maximum pulling force of the selected artificial muscle is $630 \mathrm{~N}$. Therefore, the maximum assisted torque can be approximately $75.12 \mathrm{Nm}$, which is much larger than the requirement set in the previous section.

Insert Figure 2

A small piece of 2-mm-thick cow leather was glued on the shoe, and three right angle brackets were attached on the leather from the middle to the outer side of the forefoot. The cow leather prevents deformation caused from pulling of artificial muscle, and the three placements of brackets provide choices for simultaneous extroversion assistance caused from different extents of introversion posture $[2,3]$. It is extremely simple and quick to put the lower-limb part on the user's body. First, the artificial muscle is attached to the knee pad with magic tape. Second, the spring is hooked on the bracket. Last, the cable between the artificial muscle and the spring is adjusted for a sufficient dorsiflexion assistance angle. The angle was set between and the range of motion for the user of the dorsiflexion posture referenced from a healthy people's dorsiflexion angle [19]. The total time for setting the lower-limb part on 
a healthy person would not be longer than 2 minutes. It is worth noting that dorsiflexion movement is assisted with full contraction of the artificial muscle, and the assisted dorsiflexion angle was set under artificial muscle's full contraction condition. Therefore, this physical constraint of contraction length makes our system much safer than motor-actuated robotic devices. Meanwhile, excluding the right angle brackets, none the components of lower-limb parts are rigid, and the artificial muscle assists dorsiflexion movement by directly pulling the forefoot. This design not only realizes an extremely low-weighted prototype but also prevents possible joint mismatch and discomfort issues for exoskeleton-type devices [24].

\section{B. Waist Part}

The waist part consists of a microcontroller with an embedded wireless module (nRF51822, Nordic Semiconductor, Norway), two solenoid valves for carbon dioxide gas injection and release to the artificial muscle (BV214A, Mac Valves Inc., USA), a rechargeable battery compatible with medical applications (RRC1120, RRC power solutions $\mathrm{GmbH}$, Germany), and a portable gas cylinder containing $74 \mathrm{~g}$ of carbon dioxide gas. The microcontroller unit with the battery was attached on the waist band with magic tape, and the valves and gas cylinder were placed in separate back pockets. Gas is transmitted through 6-mmdiameter tubes.

The battery is able to provide power to the system consecutively for approximately 3.5 hours, which is apparently sufficient even for a chronic stage patient. The carbon dioxide gas cylinder is enough for at least 50 full contractions of the artificial muscle through practical tests; that is, at least 50 dorsiflexion movements could be assisted through the air source of one portable cylinder. The step length of a subacute stroke patient is approximately $0.3 \mathrm{~m}$ [25]. With our previous experience, even some chronic patients require rest after one $10-\mathrm{m}$ level ground walking. It contains approximately 13 swing phase dorsiflexion movements, which means the air source from one gas cylinder should sufficient. The cylinder can be replaced with a new one when the patient rests.

The setting of the waist part on users is also simple. Securing the part to the waist with magic tape, activating control unit, and connecting the tube to the artificial muscle should take less than 30 seconds. Therefore, the total setup time on a user for the whole system was less than 2.5 minutes by adding the setup times of the lower-limb part and the waist part. This meets the requirement for setup time. However, the current result was acquired from healthy people. The actual setup time on subacute stroke patients will be monitored in a future pilot study.

\section{System Configuration and Control Method}

Fig. 3 shows the configuration of our high-dorsiflexion assistive system. The microcontroller received pressure sensor data through wireless communication and determined the opening and closing of the solenoid valves for air injection and release. The air source was provided by the carbon dioxide gas cylinder to the artificial muscle through solenoid valves. 
Fig. 4(a) depicts the control flow of our system. The artificial muscle contracts when no foot pressure is detected, which means dorsiflexion support is activated when the gait phase transits from the stance to the swing phase. It has to be noted that only one pressure sensor was placed on the inner forefoot side of the insole, so the artificial muscle does not extend until the mid-stance phase when foot flat occurs. Heel rocker function, the resistive dorsiflexion mechanism in the loading response phase of gait, is assisted by the tension spring, as shown in Fig. 4(b) [21]. This mechanism prevents the requirements for suitable assistance timing and speed for heel rocker function and enables a simple control method by merely monitoring the pressure sensor. Although numerous complicated control strategies have been developed for assisting gait rehabilitation, it is agreed that a simple control method is suitable for practical clinical application [13].

Insert Figure 4

\section{Experimental Design}

The purpose of this experiment is to verify the actual intervention effects on users' physiological and kinematic characteristics with the developed high-dorsiflexion assistive system. Ethical approval of this experiment was granted by the Ethics Committee of Waseda University.

\section{A. Participants with Dorsiflexion Restriction}

Six healthy and young people without gait disabilities participated in the experiment. Simple information of the participants is shown in Table 1. It is important to conduct trials before clinical applications, especially for subacute patients who require more safety concerns. To make participants gait characteristics close to those of stroke hemiplegic patients, they were requested to wear a dorsiflexion movement-restricted ankle-foot orthosis, as illustrated in Fig. 5. This orthosis applies a tension spring above the back of the calf with a coefficient of $10.39 \mathrm{~N} / \mathrm{mm}$. The spring was set at nominal length when the orthosis was at an angle of least of plantarflexion posture. Thus, a person who wears this orthosis should exert more dorsiflexion torque than usual and the decreases in MTC and compensatory movement should reflect those of stroke patients.

Table 1. Characteristics of the experiment participants 


\begin{tabular}{|lllll|}
\hline & Sex & Age & Height $(\mathrm{cm})$ & Weight $(\mathrm{kg})$ \\
\hline A & Male & 30 & 172 & 73 \\
\hline B & Male & 26 & 175 & 75 \\
\hline C & Male & 24 & 174 & 62 \\
\hline D & Male & 25 & 172 & 67 \\
\hline E & Male & 23 & 180 & 66 \\
\hline F & Male & 25 & 170 & 80 \\
\hline
\end{tabular}

Insert Figure 5

\section{B. Experimental Setting $\underline{s}$}

Interventions were conducted on the right legs for all participants. They were requested to walk $5 \mathrm{~m}$ six times under all the following three conditions with their own preferred speed:

a) Normal walk (NOR): The participants wore the orthosis, but without restriction of dorsiflexion movement.

b) Dorsiflexion restriction (DFR): The participants wore the orthosis, and the spring was applied for dorsiflexion movement restriction.

c) Assistance (AST): The participants wore the orthosis with dorsiflexion movement restriction, and the high-dorsiflexion assistive system was applied to support dorsiflexion in the swing phase.

The sEMG data of the tibialis anterior muscle were collected with the Trigno ${ }^{\mathrm{TM}}$ Wireless System (Delsys Inc., USA) with a sampling rate of $2000 \mathrm{~Hz}$. Meanwhile, the gait kinematic data were recorded by the motion capture system (MAC3D, NAC Inc., Japan) with a sampling rate of $100 \mathrm{~Hz}$. The marker set of this experiment is shown and explained in Fig. 6. Foot pressure data were also collected by force plates (Advanced Manufacturing Technology Inc., USA) to determine the stance and swing phases of gait.

Insert Figure 6

\section{Evaluations}

The physiological characteristics were evaluated with the collected SEMG data and were analyzed by the software EMGworks (Delsys Inc., USA). The data were filtered through an infinite impulse response bandpass filter with cut-off frequencies equal to $100 \mathrm{~Hz}$ to $400 \mathrm{~Hz}$. The filtered data were then processed with mean absolute value calculation, and the index was accessed by integrating the processed data during ankle dorsiflexion movement in the swing phase. This index evaluates the extent of self-activating 
dorsiflexion movement under each condition. For realization of full dorsiflexion assistance for passive gait training, a small sEMG signal during our system's assistance was expected.

For kinematic characteristics, three aspects of evaluation indices were determined. The first aspect was the direct effect on ankle kinematics. As Fig. 7(a) shows, the ankle angle was calculated with the markers RVMH, RANK, and RKNE. Dorsiflexion angle was defined as the minimum ankle angle in the swing phase, and improvement of dorsiflexion angle in AST condition compared with DRF condition was expected. The other aspects were stumbling risk and compensatory gait patterns. The stumbling risk was evaluated with MTC and registered from the minimum height of the RTOE marker in the swing phase of gait, which is also shown in Fig. 7(a). The compensatory gait patterns were divided into two types: coronal plane and sagittal plane. For compensatory gait patterns in the coronal plane, as Fig. 7(b) indicates, circumduction gait was frequent for stroke patients. The lateral pelvis tilt angle, calculated with the angle between the floor and vector from the markers LASIS to RASIS, and swing width , calculated with the horizontal length of marker RTOE in the swing phase, were evaluated. For compensatory gait patterns in sagittal plane, as shown in Fig. 7(a), excess hip flexion was frequent for stroke patients. Since an increase in knee height is an obvious sign of excess hip flexion, RKNE marker's height in the swing phase was evaluated. It was expected that either a small MTC or inclination of the compensatory gait pattern would be revealed while ankle dorsiflexion was restricted, and improvement could be observed during assistance using our highdorsiflexion assistive system.

Insert Figure 7

\section{Results}

Due to differences of effects from dorsiflexion restriction orthosis and a high-dorsiflexion assistive device, the evaluation indices for individuals instead of overall averages were analyzed. The indices of all conditions were analyzed with one-way ANOVA, with significant difference set as [26]. If significant differences were observed, Bonferroni t-Test was applied as a post hoc test, and the significant difference between two of the conditions was set as [27]. The results for all indices are presented with box and whisker plots (Figs. 8-13). They show means, standard deviations, and ranges corresponding to each data set.

Insert Figure 8-13

The results of one-way ANOVA for the physiological characteristic index showed significant differences for all participants. As Fig. 8 shows, the processed sEMG results were significantly higher in the PAR condition compared with the NOR condition. The results for the AST condition were significantly lower than those in the PAR condition, and even lower than in the NOR condition for 5 of 6 participants.

For ankle kinematics, the results of one-way ANOVA indicated significant differences for all participants. Fig. 9 shows that ankle angles in the PAR condition compared with the NOR condition showed different results, which could result from different voluntary dorsiflexion torques for each individual. Compared 
with ankle angles in the NOR condition, those in the AST condition showed nonsignificant differences or significantly lower ankle angles for Participants A, B, C and F. Conversely, ankle angles for Participants D and $E$ in the AST condition were significantly higher than those in the NOR condition.

All MTC results showed significant differences through one-way ANOVA. However, as Fig. 10 indicates, the MTC for Participant F was the only result, as expected. The MTC significantly decreased from the NOR to the PAR condition and then significantly improved in the AST condition. For the other participants, MTC reversely significantly increased from the NOR to the PAR condition and then significantly decreased in the AST condition.

Judging from the results, all participants showed different extents of compensatory movements. Figs. 11 and 12 illustrate that participant A's lateral pelvis tilt angle and swing width significantly increased, and Participant F's lateral pelvis tilt angle significantly increased from the NOR to the PAR condition. Significant improvements were observed in the AST condition, indicating inclination of circumduction gait when dorsiflexion movements were restricted, but it improved when our system assisted. For Participants B, C, and D, compensatory movements in sagittal planes were observed with significantly large knee heights in the PAR condition compared with the NOR condition. Significant decreases in knee heights were only seen for Participants B and C, which indicated inability of improving excess hip flexion with our system's assistance for Participant D. Last, as Fig. 13 indicates, compensatory movements in both coronal and sagittal planes were shown for Participant E, with significant increases in knee height and lateral pelvis tilt angle when dorsiflexion movement was restricted, and improvement of excess hip flexion was observed when our system assisted.

\section{Discussion}

Previous studies have reported RAFO devices that have aided ankle movements in gait rehabilitation for stroke survivors [8-11]. Although some of them revealed potential effects with pilot and clinical studies, participants were mainly chronic patients. We claimed that assistance of ankle movement, especially dorsiflexion in the swing phase for ensuring MTC, should be applied on subacute patients in early-stage gait rehabilitation. In this study, a high-dorsiflexion assistive device was developed. To our knowledge, the weight of the system, especially for the lower-limb part, is the lowest compared with the previous studies. This is important for lessening the load burden for subacute patients. Moreover, the output dorsiflexion torque of our system can be approximately $75.12 \mathrm{Nm}$, which is much larger than the most frequently applied motor-driven RAFOs and shows the potential of assisting passive gait rehabilitation for subacute patients with very low dorsiflexion ability. Set-up times for robotic assistive devices are usually long, but the current setup time of our system on healthy people was not longer than 2.5 minutes. Although it showed the potential of minimizing patient burden based on the length of set-up, the actual set-up time on stroke patients will be measured in the future.

An experiment was conducted to verify the assistance effect on healthy participants with dorsiflexionrestricted orthosis. Although the results for all participants showed different patterns, they can be 
concluded as follows:

- According to the results of processed sEMG for tibialis anterior muscles, the required voluntary dorsiflexion movement was significantly decreased when supported by our high-dorsiflexion assistive system.

- All participants revealed different types or extents of compensatory movements when their dorsiflexion movement was restricted, but these compensatory movements were improved with sufficient assistance from our system.

- Results of possible excess reliance on assistance of our system were indicated.

Analysis of sEMG results for tibialis anterior muscles should be the most appropriate for evaluating whether assistance for passive dorsiflexion movement is possible. The dorsiflexion restriction orthosis applied a tension spring above the posterior side of the shank. Therefore, a larger torque than that for assisting the ankle with no dorsiflexion ability should be output by our system. Due to the large output force of the McKibben-type artificial muscle, significantly lower sEMG results were observed when assisted by our system compared with even those during normal use for most of the participants. This indicates that our system should have the potential to assist passive dorsiflexion movement in gait rehabilitation for subacute patients.

Without a sufficient ankle dorsiflexion angle in the swing phase, stroke patients tend to apply compensatory movements to ensure MTC [5-7]. Similarly, all participants of our experiment revealed compensatory movements when dorsiflexion was restricted. Participant $F$ performed one of the typical patterns for stroke patients, which is inclination of both compensatory movement and smaller MTC when ankle dorsiflexion movement was restricted. For other participants, however, higher MTC results were shown when ankle dorsiflexion movements were restricted. Although quantification was not possible, we believe this compensation results from excess extents of circumduction gait and excess hip flexion movements by the healthy participants. The compensatory movements of all participants improved when they were assisted by our system, excluding Participant D. It should be noted that although the ankle angle of Participant E was significantly higher in the AST condition than that in the NOR condition with post hoc analysis, the difference of angles was only approximately ( for NOR and for AST). This assistance was still sufficient to realize improvement of excess hip flexion inclination.

Excessive reliance on robotic assistance devices was reported and discussed in related research [28]. The result of Participant $D$ is very likely to be an example of this phenomenon. In fact, the assistance angle of our system on Participant D was set incorrectly. A greater than increase of the ankle angle (i.e., a decrease of dorsiflexion movement) from the NOR to the AST condition was shown. This should indicate insufficient assistance from the system. However, the processed sEMG result of Participant D in the AST condition was still significantly lower than that in the NOR condition. It was suspected that the participant relied on our system's support even though the assisted ankle angle was insufficient. Thus, the excess hip flexion inclination for Participant $D$ was also not improved. This result not only indicated a negative effect of setting the wrong assistance angle but also suggested the requirement of noticing an excess 
reliance on robotic devices that might decrease the efficiency of rehabilitation. Moreover, a significantly lower knee height in the AST condition compared with the NOR condition was also shown for some participants. Thus, it should be noted that motor assistance to one joint could also lead to a decrease of voluntary effort from other joints.

With dorsiflexion-restricted orthosis, compensatory movements could be revealed with healthy people as participants. Although this was not able to simulate all the pathological gait patterns resulting from ankle disability, the potential of ensuring correct gait learning with our high-dorsiflexion assistive system was shown. The experience of the present study should be a reliable reference for a future pilot study on stroke patients.

It has to be noted that targets of the current high-dorsiflexion assistive system are subacute stroke patients; the system might not be suitable for chronic patients with comparatively higher gait abilities. The importance of full assistance for repetitive passive movements in early-stage rehabilitation has been widely known $[29,30]$. According to this concept, we set the positioning of our system as providing full assistance to ankle dorsiflexion in early gait rehabilitation to ensure neural recovery and correct gait learning. Thus, the risk of maintaining a compensatory gait pattern in the later recovery stage could be expected to be low. Moreover, numerous studies have emphasized the importance of patients' voluntary efforts during the later stage of rehabilitation [31,32]. Without sufficient challenges in rehabilitation, no significant advantages could be observed for robot-assisted rehabilitation over conventional methods. Although currently developed systems set clear targets for subacute patients, we are also looking forward to developing assist-as-needed control strategies for patients with different levels of disability. Thus, the applicable targets for our ankle dorsiflexion assistive system could be variable.

\section{Conclusion}

In the present research, we have developed a high-dorsiflexion assistive system for supporting passive ankle movement in gait rehabilitation. The low weight of the prototype and the high dorsiflexion output torque make our prototype potentially applicable for subacute stroke patients. An experiment was conducted to verify the assistance effects of our system on healthy participants with dorsiflexion movements restricted. The results showed that with sufficient dorsiflexion assistance, significantly lower voluntary dorsiflexion movement and significant improvement of compensatory movements could be observed. This indicated the potential of ensuring passive dorsiflexion movement and preventing compensatory gait patterns during gait rehabilitation assisted with our high-dorsiflexion assistive system. The results could be reliable references for our future conduction of pilot studies on stroke patients.

\section{Abbreviations}

MTC: Minimum Toe Clearance

RAFO: Robotic Ankle-Foot Orthosis 
sEMG: surface Electromyography

NOR: NORmal (condition for experiment)

DRF: DoRsiflexion Restricted (condition for experiment)

AST: ASsisTed (condition for experiment)

\section{Declarations}

\section{Availability of data and material}

The data that support the finding so of this study are available from the corresponding author upon reasonable request.

\section{Ethics approval and consent to participate}

Ethical approval was granted by the Ethics Committee of Waseda University.

\section{Consent for publication}

Written informed consent for publication of their clinical details and/or clinical images was obtained from the patients.

\section{Funding}

This study was supported by the JSPS KAKENHI, Grant number JP26289068, and Waseda University.

\section{Authors' contributions}

$\mathrm{JH}$ constructed the study concept, designed experiments, and prepared the draft manuscript. $\mathrm{JH}$ and $\mathrm{HC}$ collected and analyzed the data. $\mathrm{HO}$ and $\mathrm{HW}$ assisted with the study concept construction and data analysis. All members verified the contents corresponding to their contributions.

\section{Acknowledgements}

The authors would like to thank Zenyu Ogawa for his support in designing the hardware. The authors would like to thank the participants for their help with the experiment.

\section{Competing interests}

The authors declare that they have no competing interests.

\section{References}


1. Feigin VL et al (2016) Global burden of stroke and risk factors in 188 countries, during 1990-2013: a systematic analysis for the Global Burden of Disease Study 2013. Lancet Neural 15(9):913-924

2. Takashima $\mathrm{N}$ et al (2017) Incidence, management and short-term outcome of stroke in a general population of 1.4 million Japanese-Shiga Stroke Registry. Circ J 81(11):1636-1646

3. Voigt M, Sinkjaer T (2000) Kinematic and kinetic analysis of the walking pattern in hemiplegic patients with drop foot using a peroneal nerve stimulator. Clin Biomech 15(5):340-351

4. Wong AM, Pei YC, Hong WH, Chung CY, Lau YC, Chen CP (2004) Foot contact pattern analysis in hemiplegic stroke patients: an implication for neurologic status determination. Arch Phys Med Rehabil 85(10):1625-1630

5. Stanhope VA, Knarr BA, Reisman DS, Higginson JS (2014) Frontal plane compensatory strategies associated with self-selected walking speed in individuals post-stroke. Clin Biomech 29(5):518-522

6. Cruz TH, Dhaher YY (2009) Impact of ankle-foot-orthosis on frontal plane behaviors post-stroke. Gait \& Posture 30(3):312-316

7. Roche N, Bonnyaud C, Geiger M, Bussel B, Bensmail D (2015) Relationship between hip flexion and ankle dorsiflexion during walking phase in chronic stroke patients. Clin Biomech 30(3):219-225

8. Shi B et al (2019) Wearable Ankle Robots in Post-stroke Rehabilitation of Gait: A Systematic Review. Front Neurorobot. doi: 10.3389/fnbot.2019.00063

9. Bae J et al (2018) A lightweight and efficient portable soft exosuit for paretic ankle assistance in walking after stroke. In: Robotics and Automation (ICRA), 2018 IEEE International Conference on, 2820-2827, IEEE

10. Tanaka E, Muramatsu K, Osawa Y, Watanuki K, Saegusa S, Yuge L (2017) Development of a Walking Assistance Apparatus for Promotion of Exercise. In: Design Engineering and Science (ICDES), 2017 The 4th International Conference on, 223-228

11. Yaskawa Electric Coporation (2001) CoCoroe AAD. http://www.e-mechatronics.com/cocoroe/aad/. Accessed 25 Aug 2020

12. Eng JJ, Chu KS (2002) Reliability and comparison of weight-bearing ability during standing tasks for individuals with chronic stroke. Arch Phys Med Rehab 83(8):1138-1144

13. Dzahir MAM, Yamamoto S (2014) Recent Trends in Lower-Limb Robotic Rehabilitaiton Orthosis: Control Scheme and Strategy for Pneumatic Muscle Actuated Gait Traners. Robotics 3(2):120-148

14. Ferris DP, Czerniecki JM, Hannaford B (2005) An Ankle-Foot Orthosis Powered by Artificial Pneumatic Muscles. J Appl Biomech 21(2):189-197

15. Park YL, Chen BR, Pèrez-Arancibia NO, Young D, Stirling L, Wood RJ, Goldfield EC, Nagpal R (2014) Design and control of a bio-inspired soft wearable robotic device for ankle-foot rehabilitation. Bioinspir Biomim 9(1):016007

16. Tondu B, Lopez P (2000) Modeling and Control of McKibben Artificial Muscle Robot Actuators. IEEE Contr Syst Mag 20(2):15-38 
17. Wang S, Sato K (2016) High-precision motion control of a stage with pneumatic artificial muscles. 43:448-461

18. Wickramatunge KC, Leephakpreeda T (2010) Study on mechanical behaviours of pneumatic artificial muscle. Int J Eng Sci 48(2):188-198

19. Perry J, Burnfield J (2010) Ankle-Foot Complex. In: Gait Analysis: Normal and Pathological Functions, 2nd edn., Slack Incorporated, New Jersey

20. Hong JC, Suzuki S, Fukushima Y, Yasuda K, Ohashi H, Iwata H (2018) Development of HighDorsiflexion Assistive Robotic Technology for Gait Rehabilitation. In: Systems, Man, and Cybernetics (SMC), 2018 IEEE International Conference on, 3801-3806, IEEE

21. Hong JC, Hayashi Y, Suzuki S, Fukushima Y, Yasuda K, Ohashi H, Iwata H (2019) Identification of Spring Coefficient for Heel Rocker Function Support Based on Estimated Dorsiflexion Torque. In: Rehabilitation Robotics (ICORR), 2019 IEEE International Conference on, 355-359, IEEE

22. Zhou Z, Sun Y, Want N, Gao F, Wei K, Wang Q (2016) Robot-Assisted Rehabilitation of ankle Plantar Flexors Spasticity: A 3-Month Study with Proprioceptive Neuromuscular Facilitation. Front Neurorobot 10(16):1-14

23. Research Institute of Human Engineering for Quality Life (1997) Japanese Body Size Data.

24. Zanotto D, Akiyama Y, Stegall P, Agrawal SK (2015) Knee Joint Misalignment in Exoskeletons for Lower Extremities: Effects on User's Gait. IEEE Trans Robot 31(4):978-987

25. Mao YR, Lo WL, Lin Q, Li L, Xiao X, Raghavan P, Huang DF (2015) The Effect of Body Weight Support Treadmill Training on Gait Recovery, Proximal Lower Limb Motor Pattern, and Balance in Patients with Subacute Stroke. Biomed Res Int 2015:175719

26. Heiberger RM, Neuwirth E (2009) One-Way ANOVA. In: R Through Excel, Springer, New York

27. Cleophas TJ, Zwinderman AH (2011) Bonferroni t-Test. In: Statistical Analysis of Clinical Data on a Pocket Calculator, Springer, Dordrecht

28. Kooij H, Koopman B, Asseldonk EHF (2008) Body weight support by virtual model control of and impedance exoskeleton (LOPES) for gait training. In: Engineering in Medicine and Biology Society (EMBS), 2008 30th Annual International Conference of, 1969-1972, IEEE

29. Morone G, Bragoni M, losa M, Angelis DD, Venturiero V, Coiro P, Pratesi L, Paolucci S (2011) Who May Benefit From Robotic Assisted Gait Training? A Randomized Clinical Trial in Patients With Subacute Stroke. Neurorehabil Neural Repair 25(7):636-644

30. Peurala SH, Airaksinen O, Huuskonen P, Jäkälä P, Juhakoski M, Sandell K, Tarkka IM, Sivenius J (2009) Effects of intensive therapy using gait trainer or floor walking exercises early after stroke. $J$ Rehabil Med 41(3):166-173

31. Cao J, Xie SQ, Das R, Zhu GL (2014) Control strategies for effective robot gait rehabilitation: The state of art and future prospects. Med Eng Phys 36(12):1555-1566

32. Lotze M, Braun C, Birbaumer N, Anders S, Cohen LG (2003) Motor learning elicited by voluntary drive. Brain 126(4): 866-872 


\section{Figures}

(a)

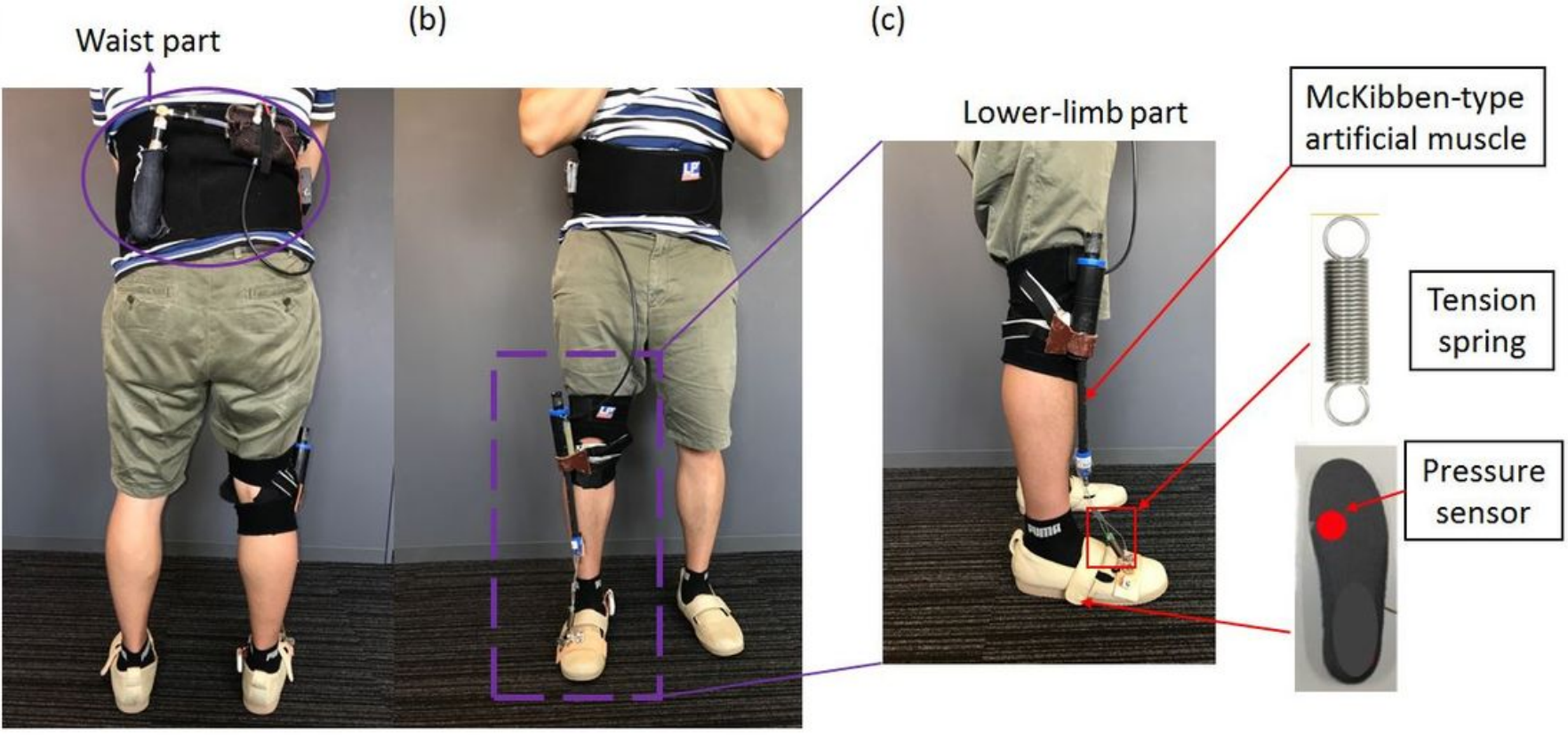

Figure 1

Appearance of the high-dorsiflexion assistive robotic technology (a) Back view of the prototype with waist part elements (b) Front view of the prototype (c) Side view of the lower-limb part with the McKibben-type artificial muscle, tension spring, and pressure sensor deployment highlighted 


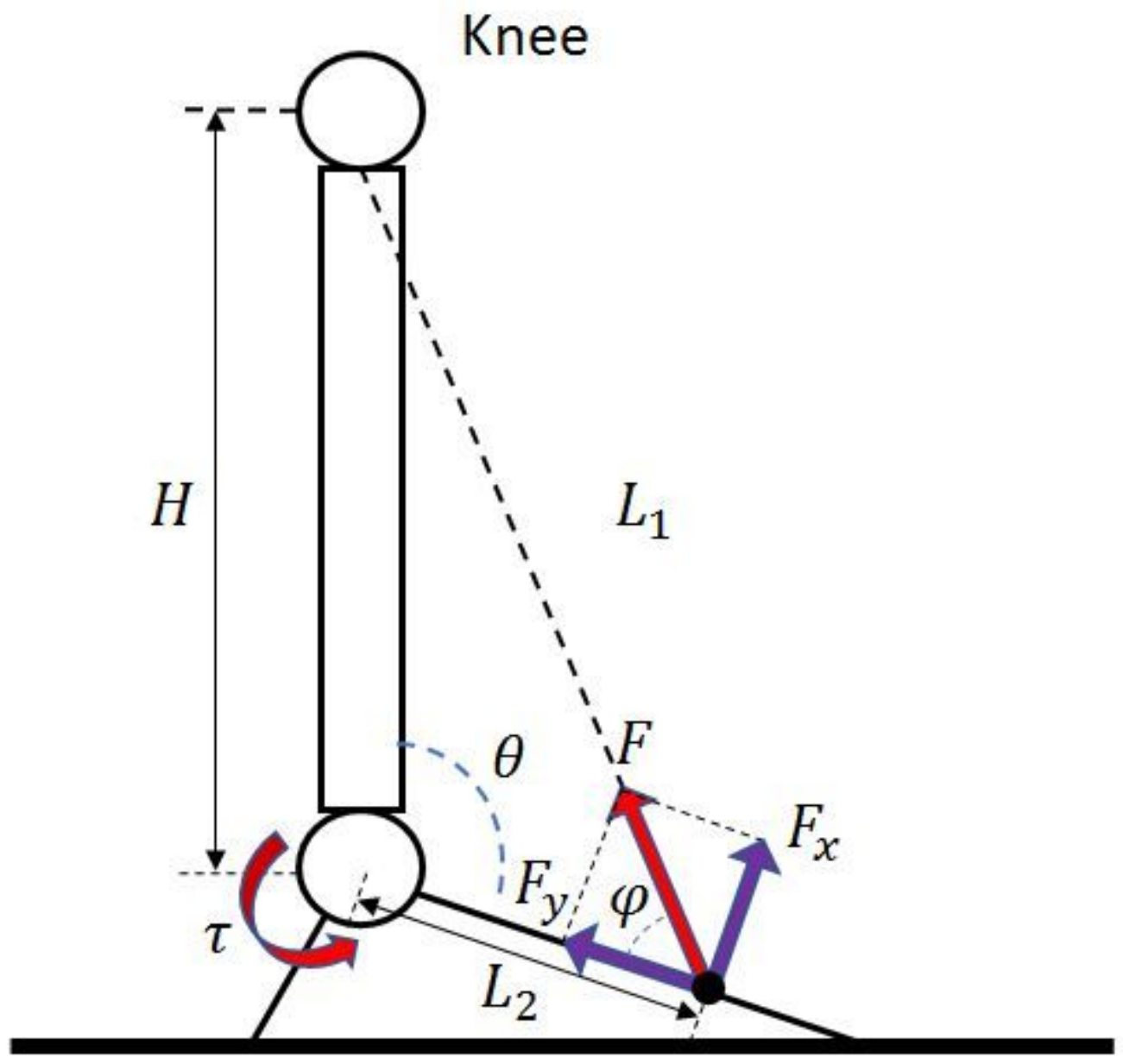

Figure 2

Simplified lower-limb model 


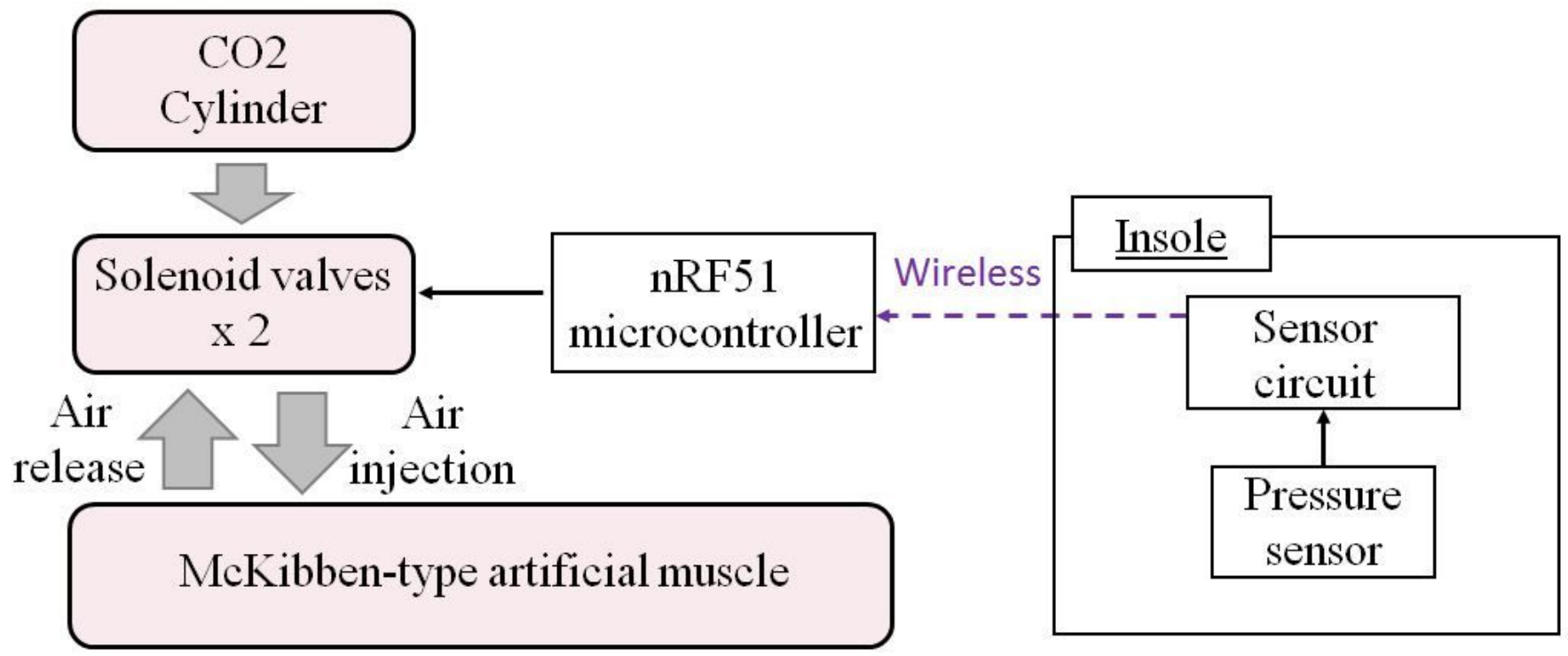

Figure 3

System configuration

(a)

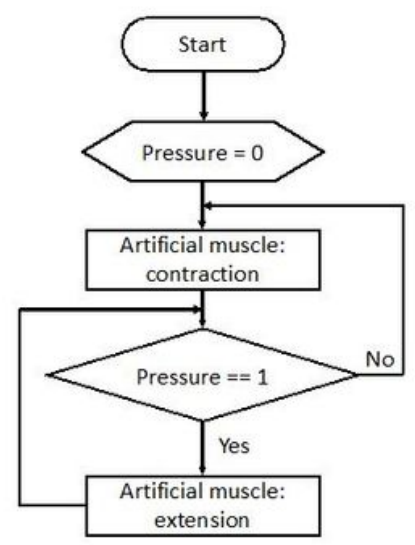

(b)

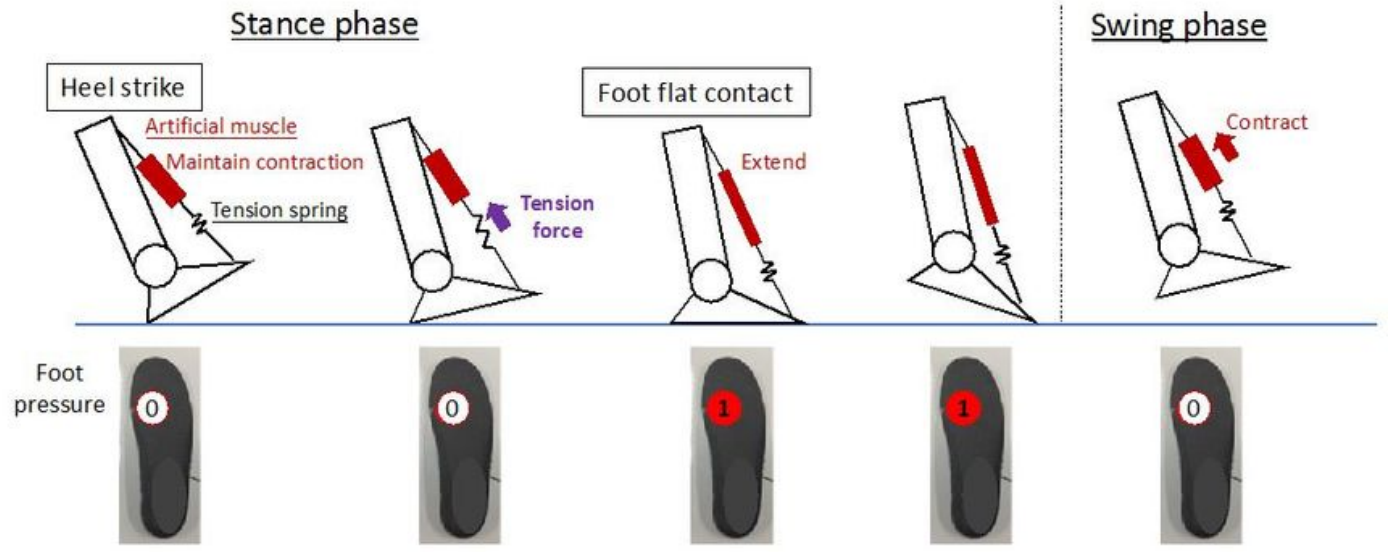

Figure 4

Control method of the system (a) Control flow diagram (b) Diagram of system control in a gait cycle 


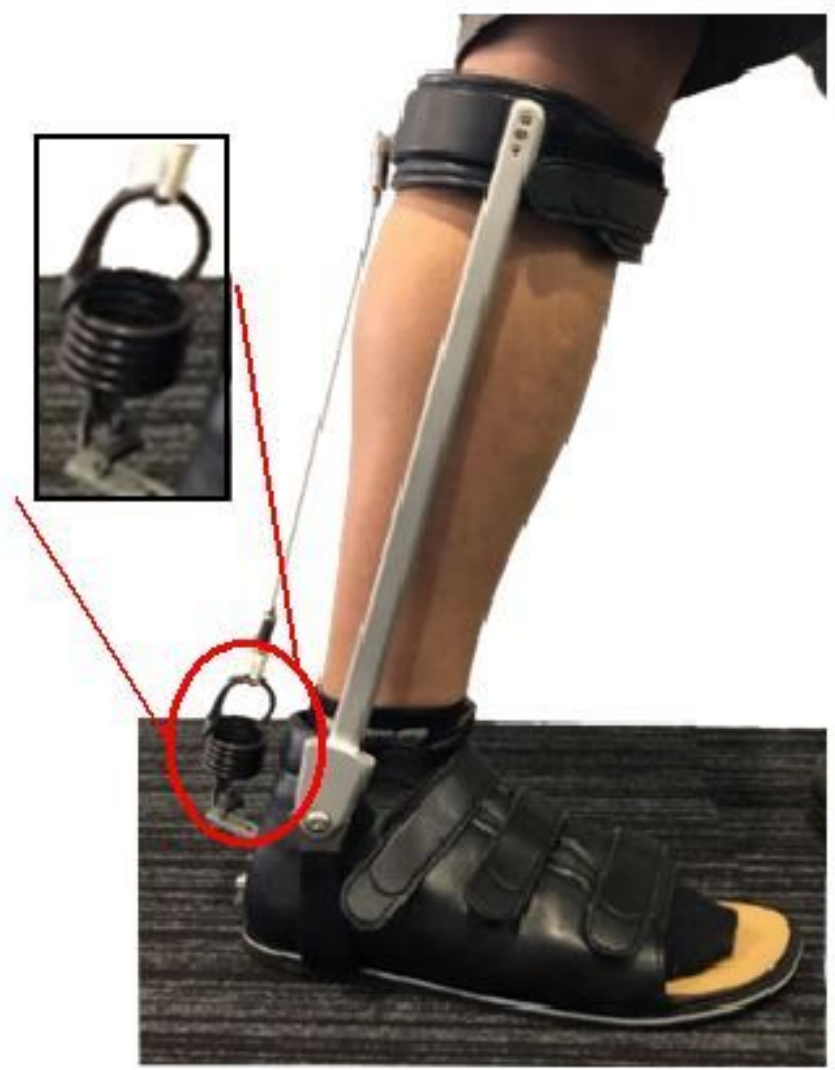

Figure 5

Dorsiflexion movement restriction orthosis 

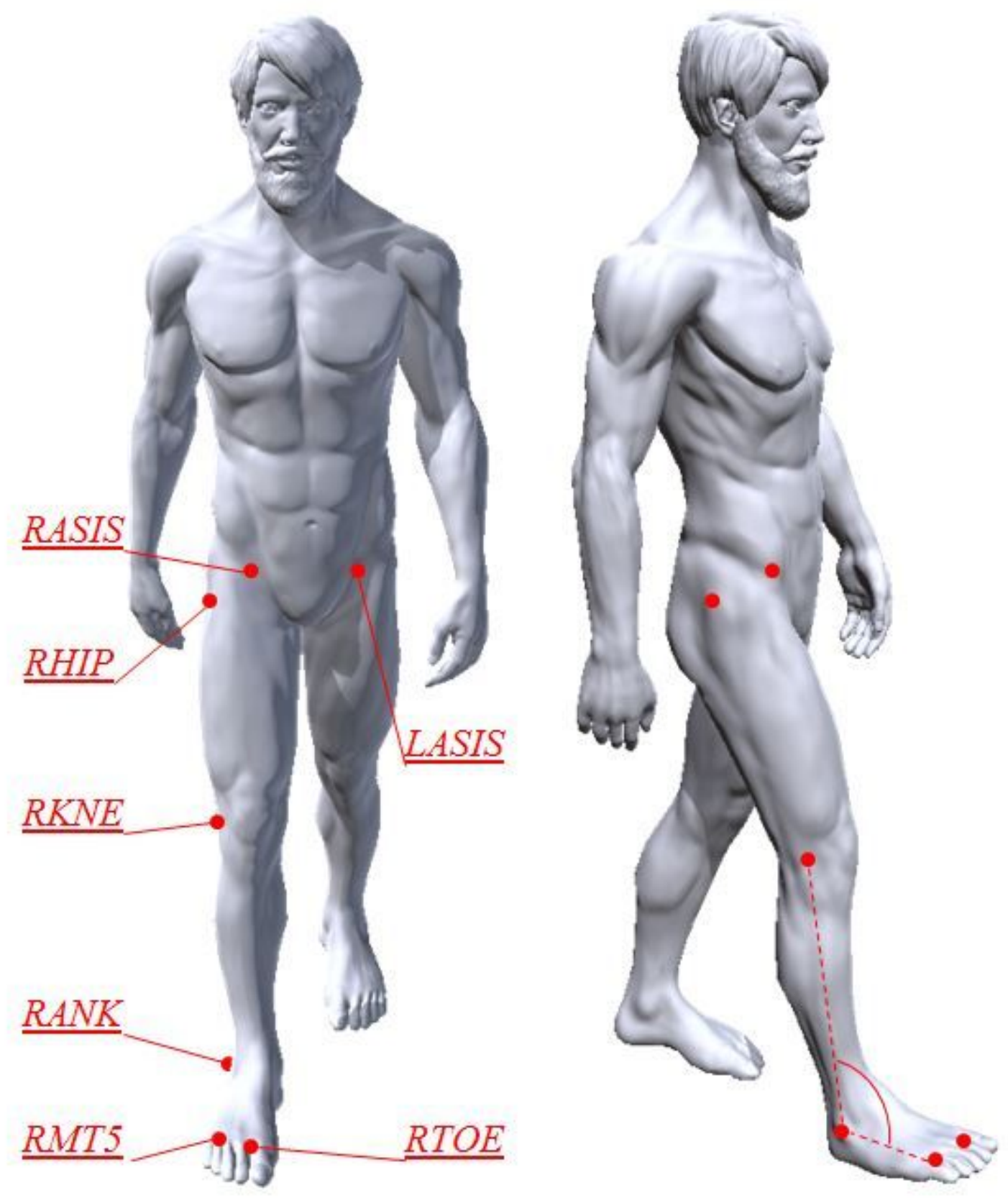

Figure 6

Placement of markers RTOE: between the 1st and 2nd metatarsal heads/RMT5: 5th metatarsal head/RANK: right ankle joint/RKNE: right knee joint/RHIP: right hip joint/RASIS, LASIS: anterior superior iliac spines for both sides 


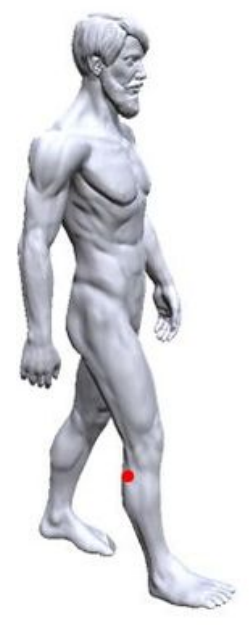

(a)

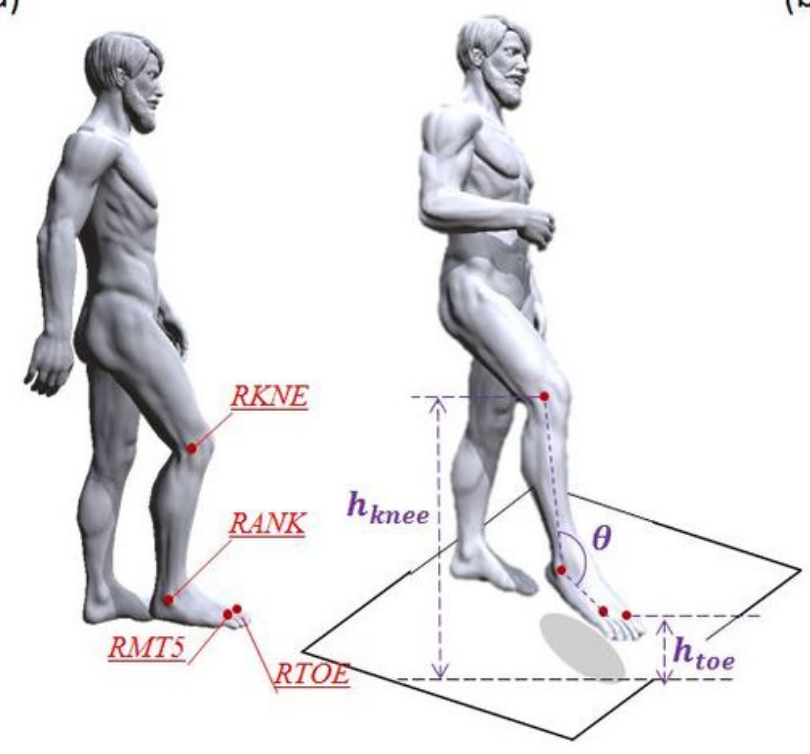

(b)

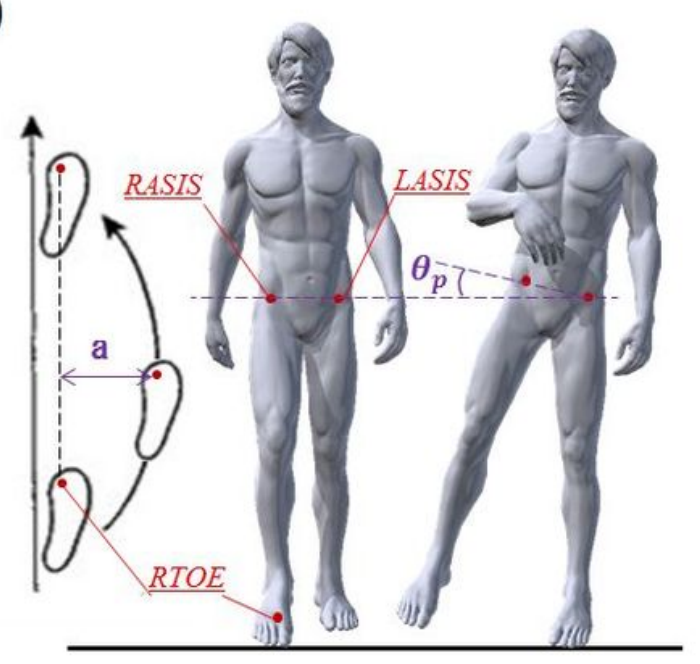

Figure 7

Spatial evaluation indices $\theta$ : Ankle angle. The dorsiflexion angle was defined as the minimum ankle angle in the swing phase h_toe: Toe height. MTC was defined as the minimum toe height in the swing phase h_knee: Knee height a: Swing width $\theta \_p$ : Lateral pelvis tilt angle 


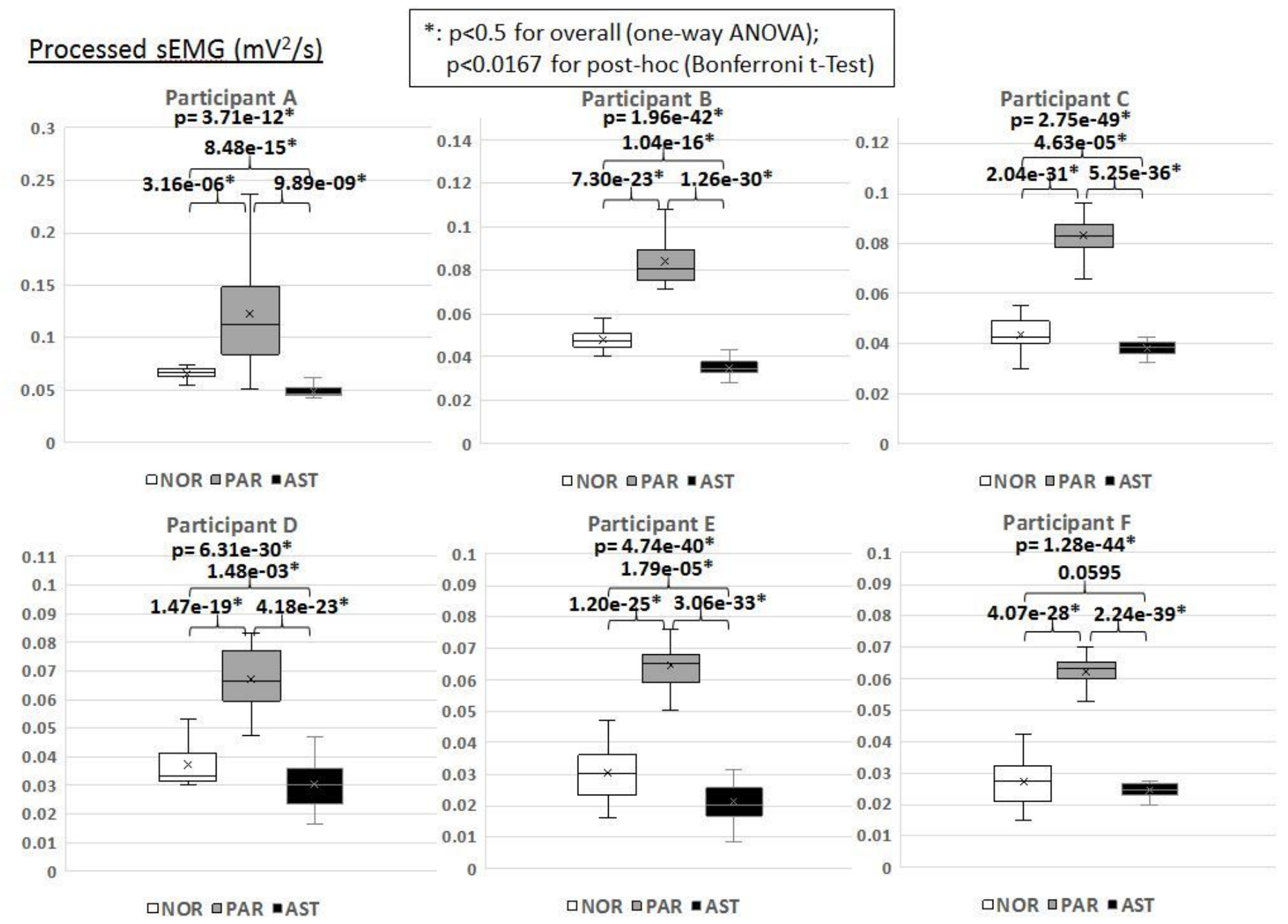

Figure 8

Results of processed sEMG 


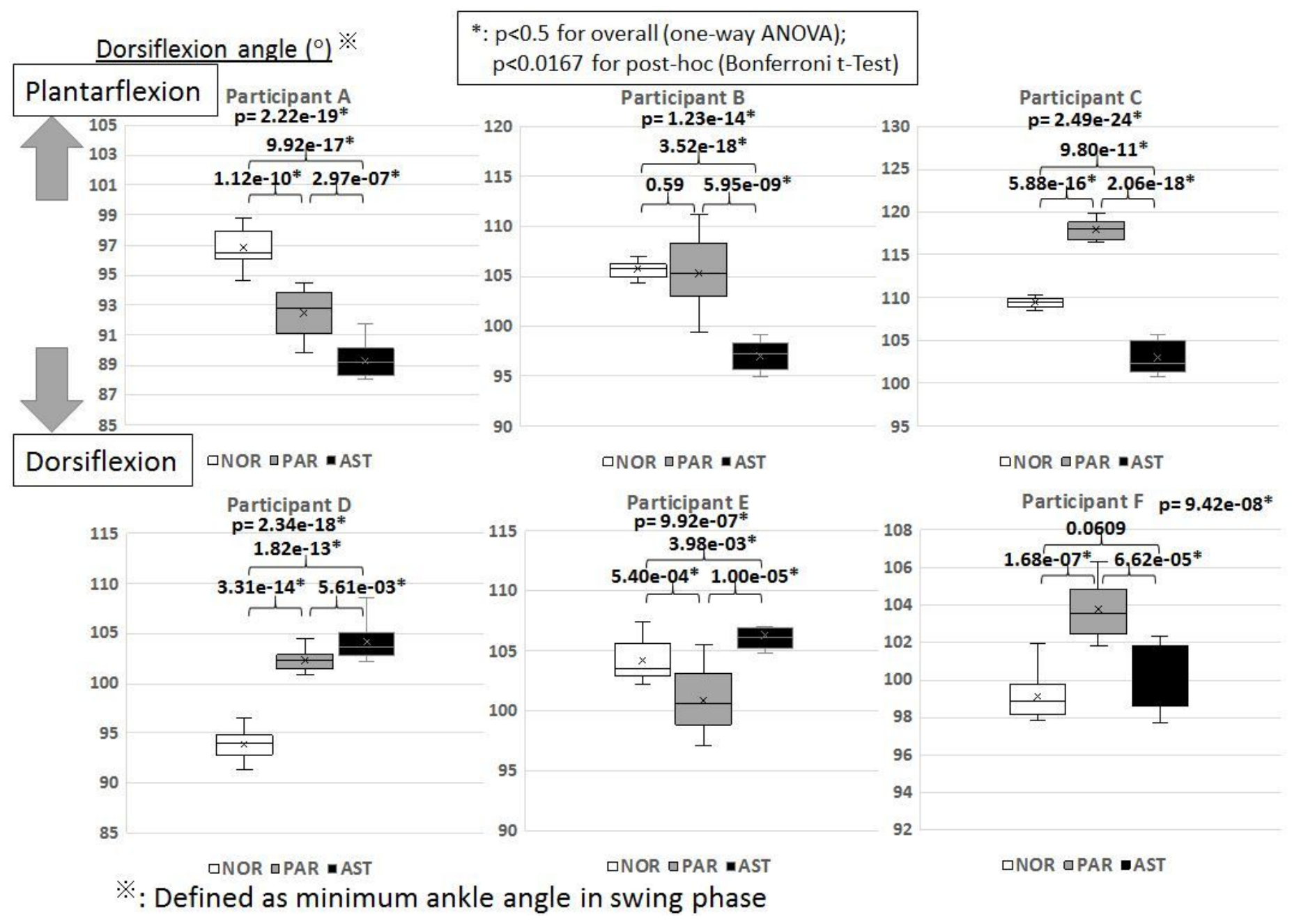

\section{Figure 9}

Results of dorsiflexion angle 


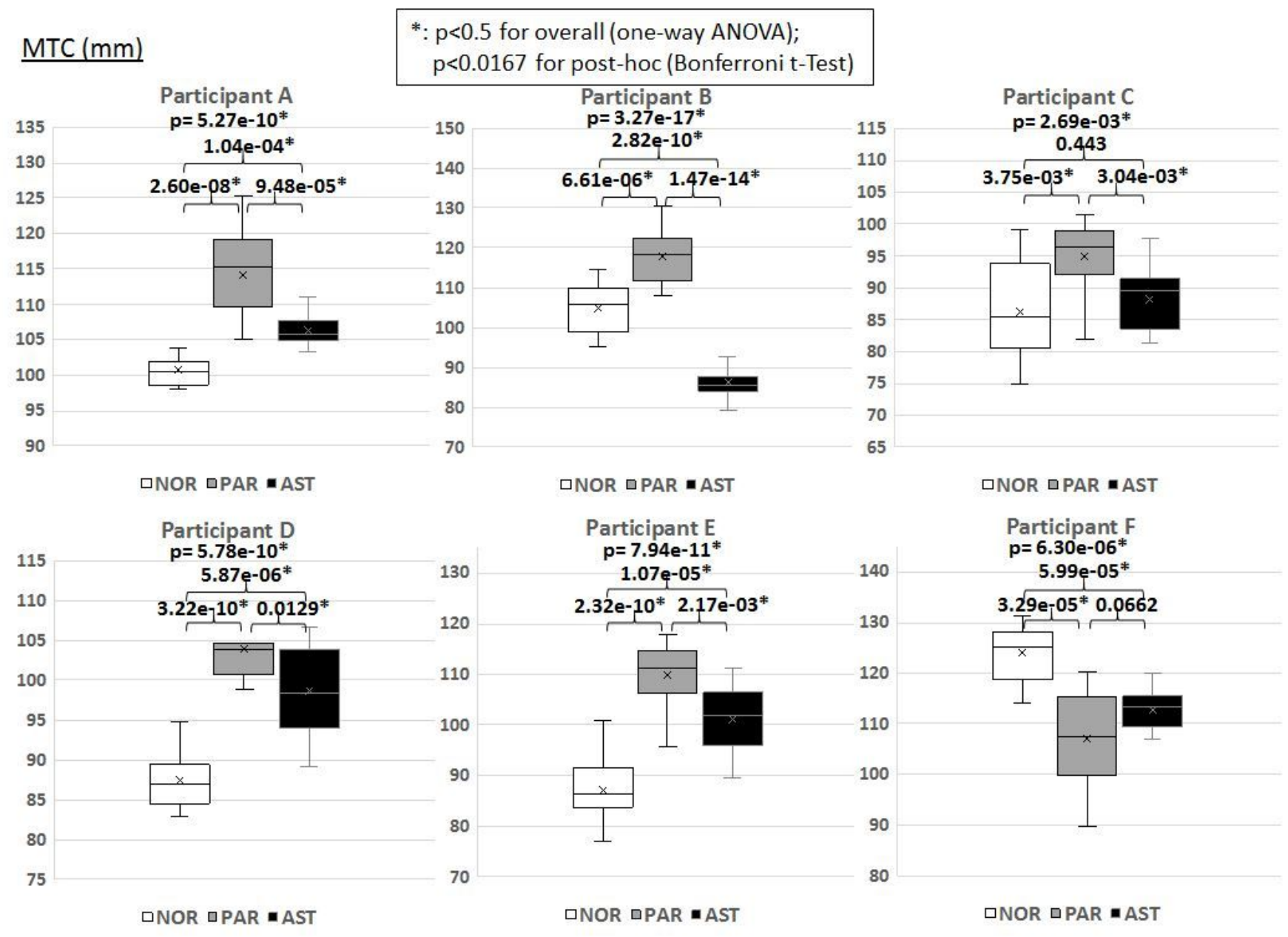

Figure 10

Results of MTC 


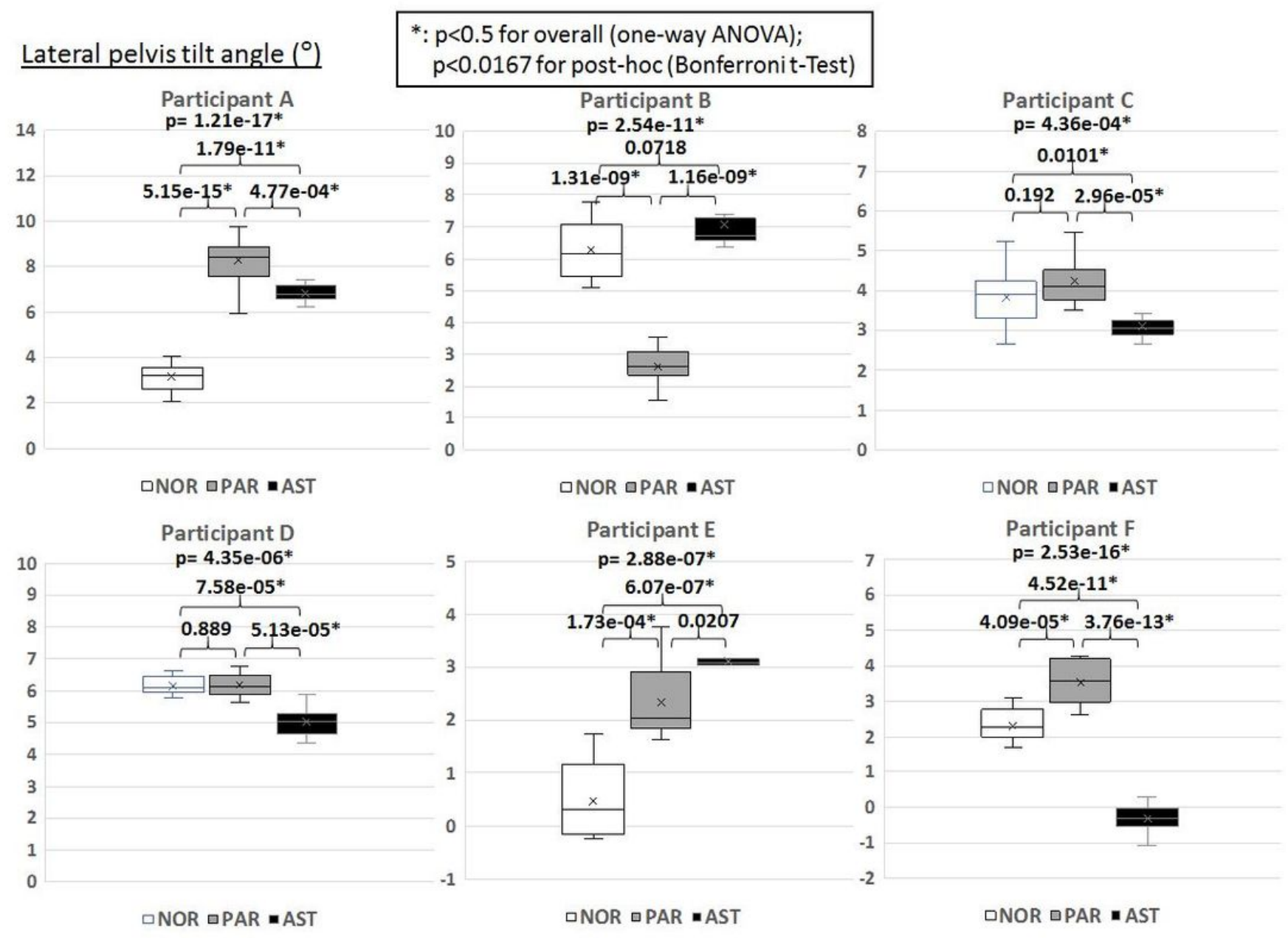

Figure 11

Results of lateral pelvis tilt angle 


\section{Swing width (mm)}

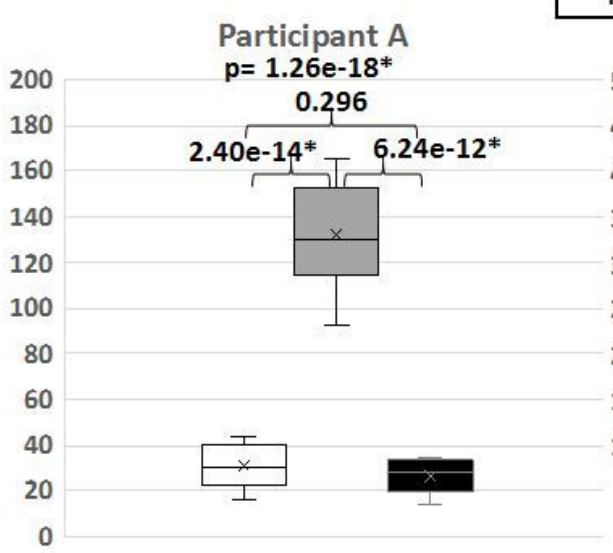

$\square N O R$ 口PAR $\backsim A S T$

Participant D

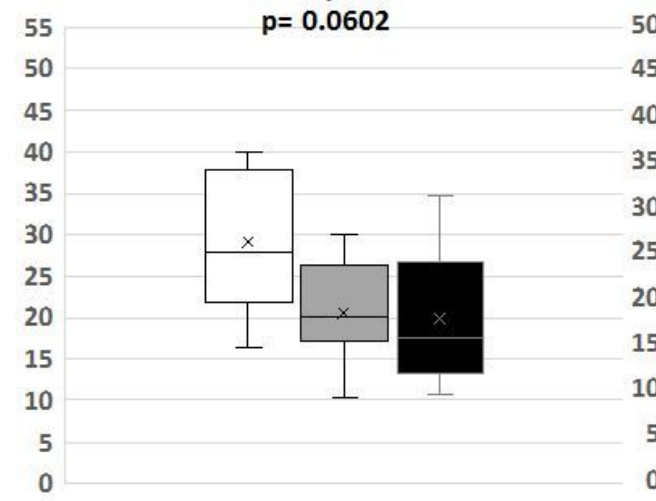

$\square N O R$ aAR ฯ AST
*: $\mathrm{p}<0.5$ for overall (one-way ANOVA); $p<0.0167$ for post-hoc (Bonferronit-Test)

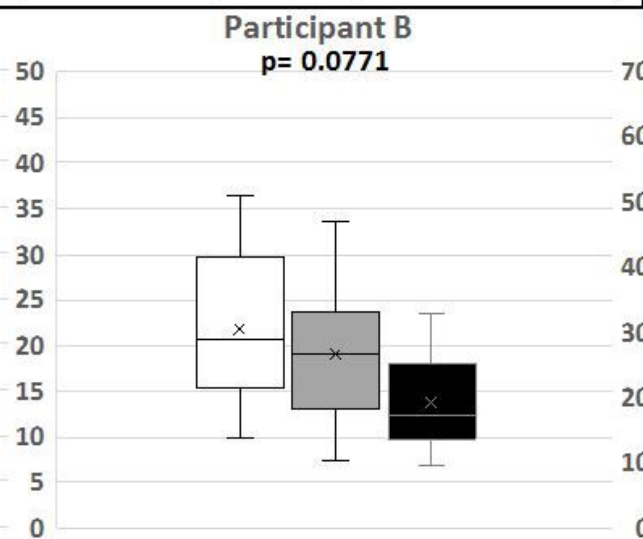

$\square \mathrm{NOR} \square \mathrm{PAR}=\mathrm{AST}$

Participant E $p=0.0526$

60

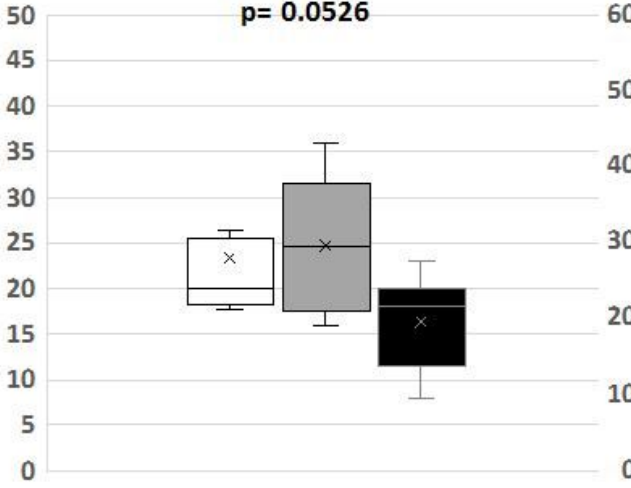

$\square \mathrm{NOR}$ 口PAR $=\mathrm{AST}$
NOR QPAR = AST

Participant F $p=0.158$

ipant C

$=4.42 \mathrm{e}-09^{*}$

2.92e-08*

$0.07166 .63 e-06^{*}$

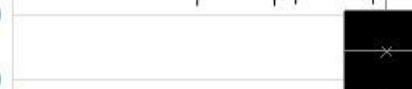

20

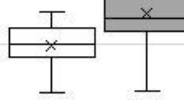

50

30

10

0

$\square$ NOR $\square$ PAR - AST

Figure 12

Results of swing width 

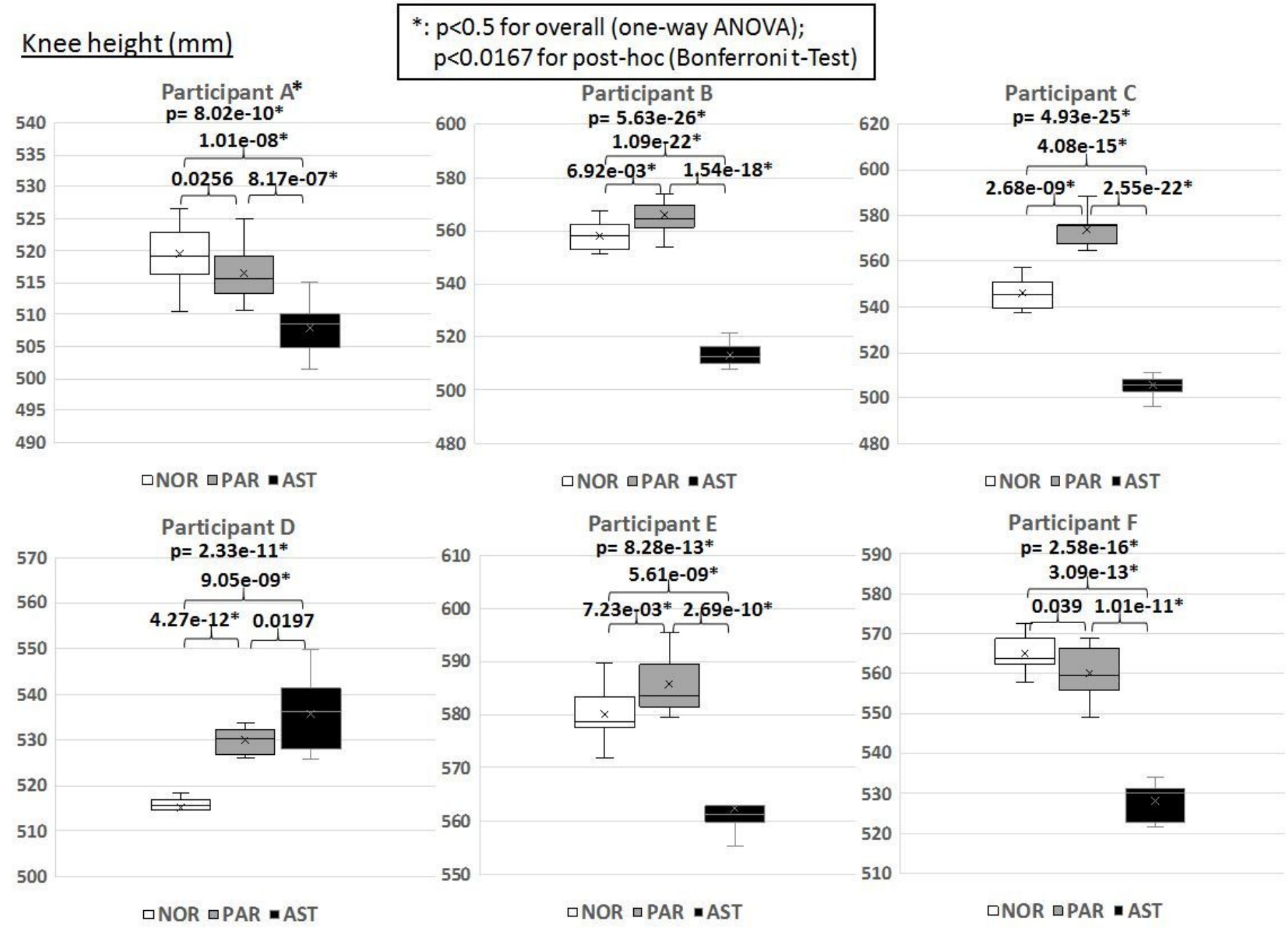